\title{
Is Less Noise, Light and Parental/Caregiver Stress in the Neonatal Intensive Care Unit Better for Neonates?
}

\author{
Rohini Venkataraman, Majeeda Kamaluddeen, Harish Amin and Abhay Lodha \\ From Department of Pediatrics, University of Calgary, Calgary, Alberta, Canada. \\ Correspondence to: Dr Abhay Lodha, Associate Professor, Department of Pediatrics, Foothills Medical Centre, Room C211, \\ 29 Street NW, Calgary, AlbertaT2N2T9, Canada.aklodha@ucalgary.ca
}

\begin{abstract}
In utero sensory stimuli and interaction with the environment strongly influence early phases of fetal and infant development. Extremely premature infants are subjected to noxious procedures and routine monitoring, in addition to exposure to excessive light and noise, which disturb the natural sleep cycle and induce stress. Non-invasive ventilation, measures to prevent sepsis, and human milk feeding improve short-term and long-term neurodevelopmental outcomes in premature infants. To preserve brain function, and to improve quality of life and long-term neurodevelopmental outcomes, the focus now is on the neonatal intensive care unit (NICU) environment and its impact on the infant during hospital stay. The objectives of this write-up are to understand the effects of environmental factors, including lighting and noise in the NICU, on sensory development of the infant, the need to decrease parental and caregiver stress, and to review existing literature, local policies and recommendations.
\end{abstract}

Keywords: Developmental disabilities, Environment, Neonate, Noise pollution.

$\mathrm{T}$ he immature organ systems, including the brain, of a preterm infant nursed in the neonatal intensive care unit (NICU) are vulnerable to any change in the NICU milieu [1]. Motherinfant contact is essential for the development of infants' physiological systems such as handling and regulation of stress, autonomic functioning and sleep patterns [2,3]. However, prolonged stay in the NICU does not allow a preterm infant the full benefit of mother-infant interaction.

In the past, it was assumed that newborns were not sensitive to the environment, and hence not capable of interaction [4]. However, it is now evident that neonates, in addition to differentiating between various stimuli and showing preference for certain stimuli, are also capable of responding to touch. Unusual sensory experiences due to frequent procedures, noise, excessive light in the NICU and prolonged mechanical ventilation pose a risk to the developing brain of the preterm infant, and adversely affect long-term neurodevelopmental outcomes [5]. Thus, the physical environment of the NICU is very important for the continued normal development of preterm infants. The NICU environmental factors influencing long-term developmental outcomes are outlined in Fig. 1.

Human neurosensory development is believed to follow a particular sequence [6]. The first sensory system to develop is touch, followed by kinesthetic, chemosensory, auditory and lastly the visual system [7]. An atypically timed or an extremely intense stimulation of one sensory system may not only affect the normal development of that system, but may also affect other systems [8].

\section{NOISE}

Differentiation of hair cells begins at 10-12 weeks of gestational age (GA). At 25-29 weeks GA, the auditory system starts to function and tonotopic columns are formed, which are essential for development of complex language and musical skills [9].

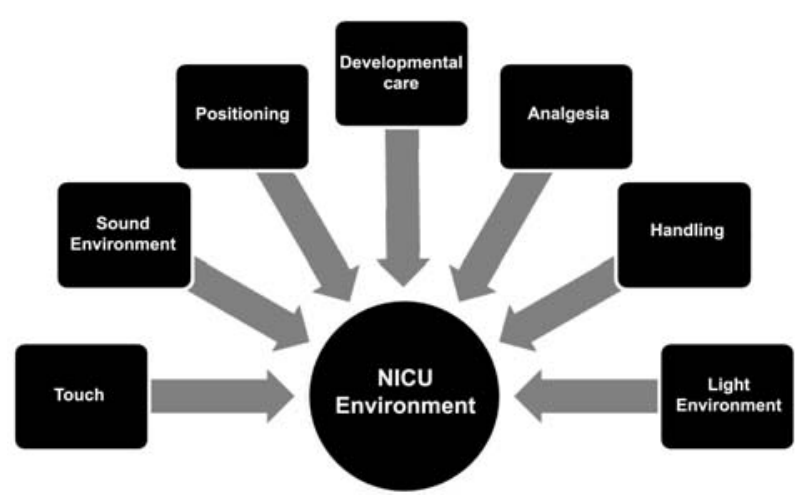

Fig.1 Environmental factors in neonatal intensive care influencing long-term developmental outcomes. 


\section{BOX 1 Stages in Auditory DeVelopment}

Genetic Endowment (15-20 weeks GA): Basic structures are formed based on genetic coding.

Endogenous Stimulation (20-22 weeks GA): Regular nerve cell activity in the spiral ganglion forms synchronous waves which help in axonal growth and formation of synapses with relay nuclei.

Activity-dependent Processes (28-29 weeks GA to early months of life): Hair cells and their cochlear connections start tuning for specific sound frequencies.

\section{GA: gestational age.}

Stages in auditory development [6] that can be blocked by exposure to low frequency noise (70-80 dB) and epigenetic factors are shown in Box 1. As outer hair cells, which receive feedback from brainstem and modulate sounds are functional only closer to term, it is difficult for the preterm infants to modulate an intense signal such as sound applied directly to the abdomen over the uterus [10]. Thus, a loud sound $(\geq 60 \mathrm{~dB})$ in utero or in NICU at 25-26 weeks gestation can cause changes in the autonomic functioning of the fetus that affects the heart rate, oxygenation, gastrointestinal motility, blood pressure and sleep [11]. As sick infants lack the reserve and the ability to self-regulate, they are at increased risk for hypoxia and bradycardia. Recommendations for sound levels in the NICU are shown in Table I [12]. The average decibel level of sounds in the NICU varies between $50 \mathrm{~dB}$ to $140 \mathrm{~dB}$ for various activities and interactions [13].

Maternal tissues protect the fetus from most high frequency sounds and light. Maternal voice, being low frequency, is heard well and distinguished from in utero background noise. Knowing the mother's voice probably helps in mother-infant attachment process and eventual language acquisition. The fetus can hear and learn simple music or sounds common to the environment as early as
30-32 weeks of gestation [14]. Brain waves generated during rapid eye movement (REM) sleep aid in the formation of long-term synapses in the auditory cortex. These synapses facilitate the neonate's ability to learn. Hence it is essential that rapid eye movement (REM) sleep cycles are protected [15].

Fetal learning is also linked to development of fetal autonomic nervous system. Infants as early as 34-35 weeks gestation can differentiate different emotional qualities of speech, music and moods, which are stored as auditory memories in the limbic system $[14,15]$.

Noise measuring technology in NICU is now accessible. Re-echoing spaces and excessive noise are the main sources of noise production. Thus, attention to NICU design, including sound-proofing is very important. A study by Philbin, et al. [16] found greater reduction in noise production after physical space change as compared to staff behavioral change. Studies have aimed to reduce noise exposure in NICU by using earplugs and earmuffs $[17,18]$.

Few recommendations to protect sleep cycles and possibly reduce adverse effects on infant's auditory development, as described in the literature [8] are provided below:

- Pregnant women should avoid prolonged exposure to low-frequency sound above $65 \mathrm{~dB}$.

- Direct application of earphones or other soundproducing devices to pregnant woman's abdomen or infants' ears should be avoided, as it is difficult to predict the sound level at the fetal cochlea.

- The human fetus should hear mother's voice during ordinary activities of daily living during the last 6-8 weeks and music and meaningful environmental sounds during the last 10-12 weeks of gestation, in utero or in the NICU. Live interaction with parent voices at the bedside should be facilitated.

- NICUs should have regular noise assessment and a program of noise control, including curtailment of

TABLE I RECOMMENDED NICU STANDARDS FOR SOUND LEVELS*

\begin{tabular}{lll}
\hline NICU sections & $\begin{array}{l}\text { Continuous background sound and } \\
\text { operational sound should not exceed }\end{array}$ & $\begin{array}{l}\text { Transient sounds or Lmax should } \\
\text { not exceed }\end{array}$ \\
\hline $\begin{array}{l}\text { Infant rooms and adult sleep areas } \\
\text { Staff work areas, family areas and staff }\end{array}$ & hourly Leq of $45 \mathrm{~dB}$ and an hourly L10 of $50 \mathrm{~dB}$ & $65 \mathrm{~dB}$ \\
lounge areas & hourly Leq of $50 \mathrm{~dB}$ and an hourly L10 of $55 \mathrm{~dB}$ & $70 \mathrm{~dB}$
\end{tabular}

*Adopted from reference 12; Leq is the equivalent (average) noise level across a given time-period; L10: A measure of the decibel level that can be exceeded for only $10 \%$ of the time (during a specific measurement period); Lmax: The highest decibel level measured for at least 1/20th of a second duration during the specified time period. 
talking and laughing and gentle manipulation of incubator portholes.

- Monitoring equipment should be minimal, less noisy or with visual alarms, and should not be placed on incubators. Manufacturers should aim to minimize the noise levels in their products.

- 'Quiet period' should be applied whenever possible, especially during the night.

\section{LIGHTING}

Visual development begins early in fetal life, and is structurally and functionally complete around age 3 years, based on neural plasticity. Stages involved in visual development are mentioned in Box 2 [19]. Though excessive light in NICU was previously considered to be a contributor to retinopathy of prematurity (ROP) interventions for light reduction in NICU did not seem to prevent it [20-22]. The dark environment of the fetus with its tactile, kinesthetic and auditory stimuli along with mother's pattern of rest and activity [23] expose the fetus to circadian rhythms and probably synchronize the fetal clock with the external light-dark cycle. The circadian clock controls body temperature, alertness and rhythmic hormonal production (e.g. melatonin, cortisol) [23]. Whether acquiring circadian rhythms during gestation helps in timing the physiological functions of the fetus to those of the mother is currently unknown.

Circadian rhythms have been documented as early as zero to three days after birth [24]. Interrupted triggering of the fetal circadian clock due to preterm birth or exposure of the preterm infant to near darkness or continuous bright light in the NICU may negatively

\section{BOX 2 Stages in Visual Development}

Activity-independent genetically controlled and epigenetic processes: Basic structures and nonspecific connections are formed.

Activity-dependent processes:

- Endogenous spontaneous retinal wave stimulation produced only during REM sleep directs and targets retinal ganglion cell axons and organization of the visual cortex. Protecting sleep cycles is important to avoid disrupting this organization.

- Visual stimulation begins with light and onset of vision near 40 weeks.

- For continued visual development, regular visual experience requiring light on objects, color and movement is essential. impact NICU stay, sleep, feeding, growth and long-term neurodevelopment outcome.

No benefit has been seen in preterm infants $<28$ weeks gestation exposed to light-dark cycles. After 28 weeks, there is some evidence in favor of cycled lighting [12]. Morag, et al. [25], in their review on the topic, concluded that many outcomes favored cycled light compared with near darkness and continuous bright light.

Recommendations for lighting in NICU include:

- Repeated focusing on the mother's (or human) face and objects and activities within the focal distance seem to be important.

- Provide some daily exposure to light (using indirect light when possible), avoid sleep interruptions, bright and direct lights, loud noises, high doses of sedative and depressing drugs and unnecessary physical disturbing activities.

- Enhance dark periods by dimming light during 'quiet period' and protecting REM sleep.

- Be aware of the impact of overhead lighting as well as light exposure from windows and rapid, abrupt changes in lighting intensity.

- Shielding the incubator with a blanket or cover to minimize light exposure is essential.

- Phototherapy lights increase light exposure to infants in surrounding beds. Ophthalmic eye drops also cause light sensitive eyes. Therefore, bright light should be avoided.

\section{Parental Stress}

Mother-infant bonding is a major problem in the NICU [26] due to: maternal anxiety or depression, infant being ill, inability to touch and hold the infant, loss of desired parenting role, isolation and guilt about having a presumable unhealthy child, and unfamiliarity of the NICU environment with bright lights, noisy monitoring machines, ventilators, alarms and chemical odors. An important factor that determines the effectiveness of parenting role after discharge is the quality and frequency of parental involvement while in the NICU. Parenting behavior is an important link between biological risk and developmental outcome.

In a study by Turan, et al. [27], the stress scores obtained by the Parental Stress Scale were significantly lower in the intervention group (in which parents received information they requested and all their questions were answered), in comparison to the control group where parents were informed of only routine unit 
procedures. Preyde and Ardal [28] showed that mothers in the intervention group who were in a "buddy program" (individualized telephonic support for a parent by a parent who had previous NICU experience) and participated in educational group meetings had less anxiety and depression, when compared to mothers in the control group who received routine NICU services.

Family integrated care (FICare) is a new approach that aims to promote weight gain among newborns admitted to the NICU at 21 days of life and lessen stress among parents. This novel method involves the active participation of parents in the day-to-day care of their newborn while in the NICU. FICare has four main pillars: parent education, nursing staff education and support, psychosocial support and NICU environment. Parents involved in FICare have less stress and anxiety [29] with improved breastfeeding rates prior to discharge among NICU patients [30].

Hence, the following strategies should be considered:

- Assurance, consistent information and a focal person during NICU stay to answer questions.

- Parental participation in daily rounds and all decisionmaking processes for their neonates.

- Emotional and spiritual support.

- Educational booklets and an album of nursery graduates to provide hope and understanding of the NICU working environment.

- NICU tours provided by other parents and implementation of a buddy program.

- Encourage parents to provide daily routines (feed, hold, bath, etc.) in stable infants, which will help with transition of care following discharge.

- Parents should be involved in major milestones such as first gavage feed, first 100 days of age, first time out of isolette, etc).

Contributors: RV, AKL: conceptualized, carried out data collection, drafted and edited the manuscript; MK, HA, AKL: reviewed, edited and approved the final version.

Funding: None; Competing interests: None stated.

\section{REFERENCES}

1. Blackburn S. Environmental impact of the NICU on developmental outcomes. J Pediatr Nurs. 1998;13:279-89.

2. Hofer MA. Early social relationships: a psychobiologist's view. Child Dev. 1987;58:633-47.

3. Meaney MJ. Maternal care, gene expression, and the transmission of individual differences in stress reactivity across generations. Annu Rev Neurosci. 2001;24:1161-92.

4. Sehgal A, Stack J. Developmentally supportive care and
NIDCAP. Indian J Pediatr. 2006;73:1007-10.

5. Pickler RH, McGrath JM, Reyna BA, McCain N, Lewis M, Cone $\mathrm{S}$, et al. A model of neurodevelopmental risk and protection for preterm infants. J Perinat Neonatal Nurs. 2010;24:356-65.

6. Hall JW, 3rd. Development of the ear and hearing. J Perinatol. 2000;20:S12-20.

7. Lickliter R. Atypical perinatal sensory stimulation and early perceptual development: Insights from developmental psychobiology. J Perinatol. 2000;20:S45-54.

8. Graven SN. Sound and the developing infant in the NICU: Conclusions and recommendations for care. J Perinatol. 2000;20:S88-93.

9. Graven SN, Browne JV. Auditory Development in the Fetus and Infant. Newborn Infant Nurs Rev. 2008;8:187-93.

10. Gerhardt KJ, Abrams RM. Fetal exposures to sound and vibroacoustic stimulation. J Perinatol. 2000;20:S21-30.

11. Miller CL, Byrne JM. Psychophysiologic and behavioral response to auditory stimuli in the newborn. Infant Behav Dev. 1983;6:369-89.

12. White RD, Smith JA, Shepley MM, Committee to Establish Recommended Standards for Newborn ICUD. Recommended Standards for Newborn ICU Design, eighth edition. J Perinatol. 2013;33:S2-16.

13. Bremmer P, Byers JF, Kiehl E. Noise and the premature infant: physiological effects and practice implications. J Obstet Gynecol Neonatal Nurs. 2003;32:447-54.

14. Moon CM, Fifer WP. Evidence of transnatal auditory learning. J Perinatol. 2000;20:S37-44.

15. Graven S. Sleep and brain development. Clin Perinatol. 2007;33:693-706.

16. Philbin MK, Gray L. Changing levels of quiet in an intensive care nursery. J Perinatol. 2002;22:455-60.

17. Abou Turk C, Williams AL, Lasky RE. A randomized clinical trial evaluating silicone earplugs for very low birth weight newborns in intensive care. J Perinatol. 2009 29:358-63.

18. Duran R, Ciftdemir NA, Ozbek UV, Berberoglu U, Durankus F, Sut N, et al. The effects of noise reduction by earmuffs on the physiologic and behavioral responses in very low birth weight preterm infants. Int $\mathrm{J}$ Pediatr Otorhinolaryngol. 2012;76:1490-3.

19. Graven SN, Browne JV. Visual development in the human fetus, infant, and young child. Newborn Infant Nurs Rev. 2008;8:194-201.

20. Roy MS, Caramelli C, Orquin J, Uleckas J, Hardy P, Chemtob S. Effects of early reduced light exposure on central visual development in preterm infants. Acta Pediatr. 1999;88:459-61.

21. Reynolds JD, Hardy RJ, Kennedy KA, Spencer R, van Heuven WA, Fielder AR. Lack of efficacy of light reduction in preventing retinopathy of prematurity. Light Reduction in Retinopathy of Prematurity (LIGHT-ROP) Cooperative Group. N Engl J Med. 1998;338:1572-6.

22. Jorge EC, Jorge EN, El Dib RP. Early light reduction for preventing retinopathy of prematurity in very low birth weight infants. Cochrane Database Syst Rev. 2001;8:CD000122.

23. Seron-Ferre M, Torres-Farfan C, Forcelledo ML, 
Valenzuela GJ. The development of circadian rhythms in the fetus and neonate. Semin Perinatol. 2001;25:363-70.

24. Begum E, Bonno M, Obata M, Yamamoto H, Kawai M, Komada Y. Emergence of physiological rhythmicity in term and preterm neonates in a neonatal intensive care unit. J Circadian Rhythms. 2006;4:11.

25. Morag I, Ohlsson A. Cycled light in the intensive care unit for preterm and low birth weight infants. Cochrane Database Syst Rev. 2016;8:Cd006982.

26. Feldman R, Weller A, Leckman JF, Kuint J, Eidelman AI. The nature of the mother's tie to her infant: Maternal bonding under conditions of proximity, separation, and potential loss. J Child Psychol Psychiatry. 1999;40:929-39.

27. Turan T, Basbakkal Z, Ozbek S. Effect of nursing interventions on stressors of parents of premature infants in neonatal intensive care unit. J Clin Nurs. 2008; 17:2856-66.

28. Preyde M, Ardal F. Effectiveness of a parent "buddy" program for mothers of very preterm infants in a neonatal intensive care unit. Can Med Assoc J. 2003;168:969-73.

29. O’Brien K, Bracht M, Robson K, Ye XY, Mirea L, Cruz M, et al. Evaluation of the family integrated care model of neonatal intensive care: A cluster randomized controlled trial in Canada and Australia. BMC Pediatr. 2015;15:210.

30. Verma A, Maria A, Pandey RM, Hans C, Verma A, Sherwani F. Family-centered care to complement care of sick newborns: A randomized controlled trial. Indian Pediatr. 2017;54:455-59. 